Nonlinear Processes in Geophysics, 12, 863-870, 2005

SRef-ID: $1607-7946 / \mathrm{npg} / 2005-12-863$

European Geosciences Union

(C) 2005 Author(s). This work is licensed

under a Creative Commons License.

\title{
Inversion of SAR data in active volcanic areas by optimization techniques
}

\author{
G. Nunnari ${ }^{1}$, G. Puglisi ${ }^{2}$, and F. Guglielmino ${ }^{2}$ \\ ${ }^{1}$ Department of Electrical, Electronic and System Engineering, University of Catania, Italy \\ ${ }^{2}$ Istituto Nazionale di Geofisica e Vulcanologia, Sez. di Catania, Italy
}

Received: 22 November 2004 - Revised: 28 February 2005 - Accepted: 1 March 2005 - Published: 30 September 2005

\begin{abstract}
The inversion problem concerns the identification of parameters of a volcanic source causing observable changes in ground deformation data recorded in volcanic areas. In particular, this paper deals with the inversion of ground deformation measured by using SAR (Synthetic Aperture Radar) interferometry and an inversion approach formulated in terms of an optimization problem is proposed. Based on this inversion scheme, it is shown that the problem of inverting ground deformation data in terms of a single source, of Mogi or Okada type, is numerically well conditioned. In the paper, two case studies of inverting actual SAR data recorded on Mt. Etna during eruptions occurring in 1998 and 2001 are investigated, showing the suitability of the proposed technique.
\end{abstract}

\section{Introduction}

Ground deformation is a common phenomenon of active volcanoes in response to the dynamic of different kinds of magma sources. Measurable ground deformation has been observed by several authors since the early experience reported by Omori (1914), who deduced its occurrence from the changing heights of benchmarks accompanying the 1914 eruption of Sakura-jima volcano in Japan. Techniques for monitoring ground deformation have been greatly improved in recent years and at present active volcanoes are monitored not only by using terrestrial techniques (e.g. precise leveling and trilateration) but also from space, thanks to the availability of techniques such as GPS (Global Positioning System) and SAR (Synthetic Aperture Radar). Indeed, while the GPS technique is now used on a regular basis, SAR interferometry is still expanding its range of applications. With the advent of spaceborne radar systems (ERS-1/2, JERS-1,RADARSAT, ENVISAT), SAR is becoming a new tool for active tectonics by providing mm-precision surface change maps to gen-

Correspondence to: F. Guglielmino

(guglielmino@ct.ingv.it) erate DEMs. Several studies (Gabriel et al., 1995; Massonnet and Rabaute, 1993; McCloy, 1995; Massonet and Feigl, 1998; Bürgmanm et al., 2000) have shown that SAR is a powerful and promising technique for observing the Earth's surface, for topographical measurements of vast geographical areas and the measurement of changes of a surface over a pre-defined time interval (Differential Radar Interferometry). SAR systems record both amplitude and phase of the back-scattered echoes. If two SAR images from slightly different viewing angles are considered (interferometric pair) their difference (interferometric fringes) can be usefully exploited both to generate Digital Elevation Maps (DEMs), and to measure slight topographic changes such as shifts in tectonic plates, movement of glaciers and so on. Recently, SAR interferometry was also considered to monitor ground deformation in volcanic active areas (Coltelli et al., 1996; Lanari et al., 1996; Briole et al., 1997; Puglisi and Coltelli, 2001). SAR interferometry allows integrating the techniques traditionally considered to measure ground deformations in volcanic areas, such as EDM (Electronic Distance Measuring) or GPS (Global Positioning System) techniques (Gudmundsson et al., 2002; Fernández et al., 2003). The inversion problem considered in this paper consists of identifying the parameters of two types of volcanic sources: the traditional Mogi source (Mogi, 1958), whose main advantage is the limited number of parameters involved and the Okada type source (Okada, 1985), which seems more realistic to interpret eruptive phenomena in volcanic areas such as Mt. Etna, where eruptions have their origin in dykes opening from a certain depth toward the surface (Bonaccorso, et al., 2004). Both the considered types of sources are supposed to work in a homogeneous and isotropic elastic half space.

Investigations into the structural analysis of the considered models, show that the problem is an extremely non-linear one. Hence the inversion solution cannot be obtained in closed form but can be formulated as an optimization search problem. In this paper it is shown that in spite of the high degree of non-linearity, the inverse problem is well conditioned and can be unambiguously solved. An appreciable degree of 


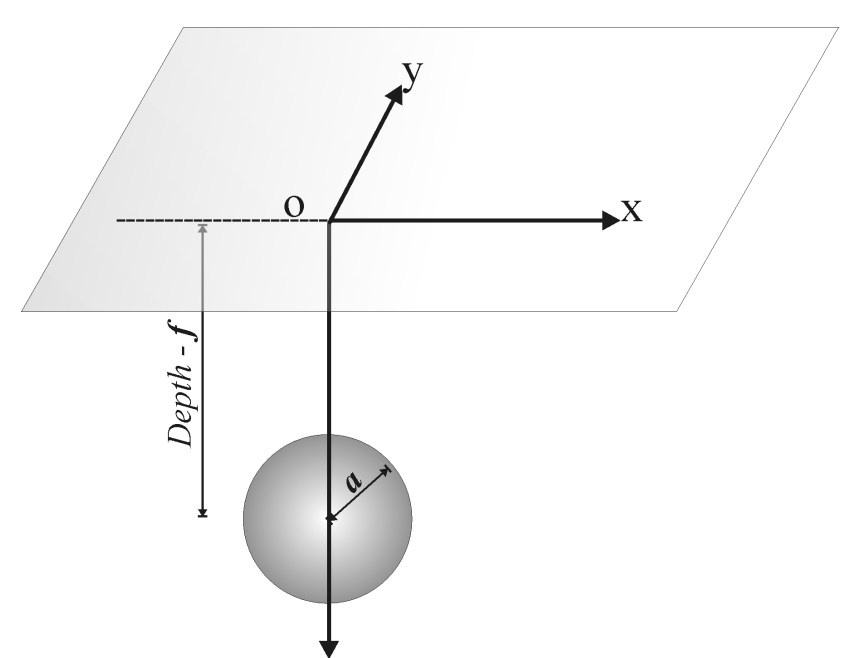

Fig. 1. The Mogi source: a spherical source with radius $a$ and acting at depth $f$.

accuracy can be obtained by using a randomized optimization algorithm such as the Genetic Optimization algorithm.

\section{Generation of SAR synthetic data}

As mentioned in the previous section, two main types of source mechanisms are considered in this paper, namely the Mogi (Fig. 1) and the Okada sources (Fig. 2). The first essentially consists of a spherical pressure source buried in a homogeneous and isotropic elastic half-space with radius $a$ and depth $f$. This source roughly simulates the presence of a magma chamber beneath the volcano surface. The second kind of source (Fig. 2) simulates a dislocation (i.e. a dyke) induced by the uprising of magma. In Fig. 2 "Depth" is the distance between the origin $O$ and the upper edge of the source; "Strike" ( $\sigma$ angle) is the orientation of the source with respect to North; "Dip" (hereafter $\delta$ ) is the angle of the dislocation plane with respect to the horizontal plane; "Length" and "Width" are the length and width of the source, respectively; $U_{1}, U_{2}$ and $U_{3}$ are the Strike-slip, Dip-slip and Tensile dislocation components, respectively.

\subsection{The Mogi model}

The original Mogi model (Mogi, 1958) was a turning point in volcanology, in that for the first time a mathematical model was presented which adequately described the deformation observed in some particular kinds of volcanoes.

The components $u_{x}, u_{y}$ and $u_{z}$ of the displacement induced by a Mogi source at a point having coordinates $(x, y$, $x$ ) have been recomputed by Sasai (1991) and are expressed by:

$u_{x}=\frac{C}{2 \mu}\left\{\frac{x}{R_{1}^{3}}-\frac{\lambda+3 \mu}{\lambda+\mu} \frac{x}{R_{2}^{3}}-\frac{6 x z(z+f)}{R_{2}^{5}}\right\}$

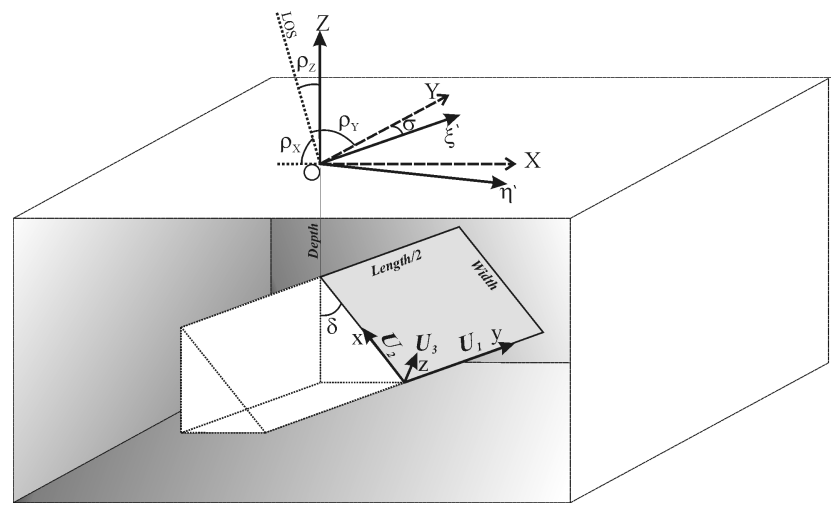

Fig. 2. Geometric representation of a rectangular fault occurring in a homogeneous and isotropic elastic half-space.

$u_{y}=\frac{C}{2 \mu}\left\{\frac{y}{R_{1}^{3}}-\frac{\lambda+3 \mu}{\lambda+\mu} \frac{y}{R_{2}^{3}}-\frac{6 y z(z+f)}{R_{2}^{5}}\right\}$

$u_{z}=\frac{C}{2 \mu}\left\{\frac{z-f}{R_{1}^{3}}-\frac{(\lambda-\mu) z-(\lambda+3 \mu) f}{(\lambda+\mu) R_{2}^{3}}-\frac{6 z(z+f)^{2}}{R_{2}^{5}}\right\}$

where:

$R_{1}=\sqrt{x^{2}+y^{2}+(z-f)^{2}}$

$R_{2}=\sqrt{x^{2}+y^{2}+(z+f)^{2}}$

$\lambda$ and $\mu$ are Lamè's constant, $C$ is the nucleus strain. Assuming that the radium is small compared with the depth $f$, the nucleus strain can be computed as:

$C=-\frac{1}{2} a^{3} \Delta P$.

\subsection{The Okada model}

Analytical expressions to describe the surface deformation due to dislocation were provided by Okada (1985) for strike slip, dip-slip and tensile fault movements. Since these expressions are rather complicated we report here the case for tensile faults only (i.e. with reference to Fig. $2, U_{3} \neq 0$ and $U_{1}=U_{2}=0$ ) since this is usually the most relevant in volcanic areas. The components $u_{x}, u_{y}$ and $u_{z}$ of the displacement are expressed by:

$$
\left\{\begin{array}{l}
u_{x}=\frac{U_{3}}{2 \pi}\left[\frac{q^{2}}{R(R+\eta)}-I_{3} \sin ^{2} \delta\right] \| \\
u_{y}=\frac{U_{3}}{2 \pi}\left[\frac{-\tilde{d} q}{R(R+\xi)}-\sin \delta\left\{\frac{\xi q}{R(R+\eta)}-\tan ^{-1} \frac{\xi \eta}{q R}\right\}-I_{1} \sin ^{2} \delta\right] \| \\
u_{z}=\frac{U_{3}}{2 \pi}\left[\frac{\tilde{y} q}{R(R+\xi)}+\cos \delta\left\{\frac{\xi q}{R(R+\eta)}-\tan ^{-1} \frac{\xi \eta}{q R}\right\}-I_{5} \sin ^{2} \delta\right] \|
\end{array}\right.
$$

where:

$$
\left\{\begin{array}{l}
p=y \cos \delta+d \sin \delta \\
q=y \sin \delta-d \cos \delta \\
\tilde{y}=\eta \cos \delta+q \sin \delta \\
\tilde{d}=\eta \sin \delta-q \cos \delta \\
R^{2}=\xi^{2}+\eta^{2}+q^{2}=\xi^{2}+\tilde{y}^{2}+\tilde{d}^{2} \\
X^{2}=\xi^{2}+q^{2}
\end{array}\right.
$$


and

$$
\left\{\begin{array}{l}
I_{1}=\frac{\mu}{\lambda+\mu}\left[\frac{-1}{\cos \delta} \frac{\xi}{R+\tilde{d}}\right]-\frac{\sin \delta}{\cos \delta} I_{5} \\
I_{2}=\frac{\mu}{\lambda+\mu}[-\ln (R+\eta)]-I_{3} \\
I_{3}=\frac{\mu}{\lambda+\mu}\left[\frac{1}{\cos \delta} \frac{\tilde{y}}{R+\tilde{d}}-\ln (R+\eta)\right]+\frac{\sin \delta}{\cos \delta} I_{4} \\
I_{4}=\frac{\mu}{\lambda+\mu} \frac{1}{\cos \delta}[\ln (R+\tilde{d})-\ln (R+\eta) \sin \delta] \\
I_{5}=\frac{\mu}{\lambda+\mu} \frac{2}{\cos \delta} \tan ^{-1} \frac{\eta(X+q \cos \delta)+X(R+X) \sin \delta}{\xi(R+X) \cos \delta}
\end{array}\right.
$$

or

$$
\left\{\begin{array}{l}
I_{1}=-\frac{\mu}{2(\lambda+\mu)} \frac{\xi q}{(R+\tilde{d})^{2}} \\
I_{3}=\frac{\mu}{2(\lambda+\mu)}\left[\frac{\eta}{R+\tilde{d}}+\frac{\tilde{y} q}{(R+\tilde{d})^{2}}-\ln (R+\eta)\right] \\
I_{4}=-\frac{\mu}{\lambda+\mu} \frac{q}{R+\tilde{d}} \\
I_{5}=-\frac{\mu}{\lambda+\mu} \frac{\xi \sin \delta}{R+\tilde{d}}
\end{array}\right.
$$

The choice between expressions (9) or (10) depends on Dip angle $\delta$. In particular, expressions (9) hold when $\cos (\delta \neq 0$, while expressions (10) when $\cos (\delta)=0$. In expressions (7) to $(10),(x, y, z)$ and $(\xi, \eta, q)$ are coordinates of appropriate reference systems, namely the former is centered at the midpoint of the bottom side of dislocation and the latter is centered on the projection on the appropriate surface of the midpoint upper side of the dislocation.

$R, \tilde{d}$ and $\tilde{y}$ are scalar quantities and, "||" represents Chinnery's notation, which is represented by the following expression:

$f(\xi, \eta)\left|\begin{array}{l}b \\ a\end{array}\right| \begin{gathered}d \\ c\end{gathered}=f(a, c)-f(a, d)-f(b-c)+f(b-d)$.

\subsection{Generation of synthetic interferograms}

In order to generate synthetic interferograms we must perform the following three steps:

1. Refer the ground deformation vector $\boldsymbol{u}=\left(u_{x}, u_{y}, u_{z}\right)$ to the geographic reference system $O(X, Y, Z$ ) (see Fig. 2). This allows to obtain a ground deformation vector $\boldsymbol{U}=\left(U_{X}, U_{Y}, U_{Z}\right)$ whose components are expressed by:

$\left\{\begin{array}{l}U_{X}=u_{x} \sin \sigma-u_{y} \cos \sigma \\ U_{Y}=u_{x} \cos \sigma+u_{y} \sin \sigma \\ U_{Z}=u_{z}\end{array}\right.$

where $\sigma$ is the "Strike" angle.

2. Project the deformation vector computed in the previous step along the satellite Line Of Sight (LOS). This is accomplished by computing the scalar quantity $U_{\rho}$ as:

$U_{\rho}=\boldsymbol{\rho} \cdot U=\rho_{X} U_{X}+\rho_{Y} U_{Y}+\rho_{Z} U_{Z}$

In expression (13) $\rho$ is the vector of cosine direction defining the satellite LOS with respect to $O=(X, Y, Z)$.

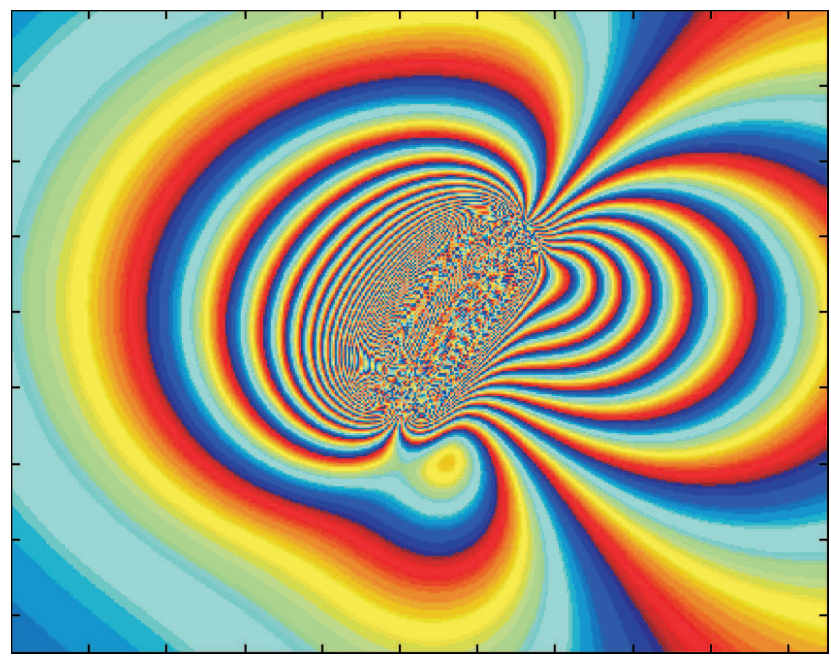

Fig. 3. Synthetic interferogram due to a dislocation having the following parameters: $\mathrm{Dip}=84.32^{\circ}, \quad$ Strike $=33.55^{\circ}$, Length $=5.03 \mathrm{~km}, \quad$ Width $=0.73 \mathrm{~km}, U_{1}=4.75 \mathrm{~m}, U_{2}=-4.92 \mathrm{~m}$, $U_{3}=2.27 \mathrm{~m}$, Depth $=0.24 \mathrm{~km}$.

3. Wrapping the quantity into the interval $[0-2 \pi]$ to calculate the fringes that characterise an interferogram, by using the relation

$\bar{U}_{\rho}=\frac{2 \pi}{\lambda} \operatorname{frac}\left(\frac{U_{\rho}}{\lambda}\right)$,

where $\lambda$ is the principal wave length emitted by the satellite antenna, that is $28^{*} 10^{-3} \mathrm{~m}$ in the case of ERS2 , and $f r a c()$ is the fractionary part. An example of a synthetic interferogram generated by a dislocation source is reported in Fig. 3.

\section{Inversion of ground deformation data by using Mogi or Okada sources}

Ground deformation models, such as the Okada model, are highly non-linear and characterized by a great number of parameters. Therefore, the inverse numerical problem could be difficult to solve by applying methods based on the linearization approach. On the other hand, a trial and error procedure may take a long time and the convergence and the quality of the results might not be accurate enough.

\subsection{Formalization of the inverse modelling problem}

The inverse modeling problem can be formalized as follows. Consider a nonlinear input-output mapping describing the so-called direct problem, expressed by the functional relationship:

$\boldsymbol{d}=f(\boldsymbol{x})$

In Eq. (15) $\boldsymbol{x}$ represents the vector of model parameters (i.e. the volcanic source) and $\boldsymbol{d}$ represents the corresponding data 
vector (i.e. the expected or measured ground deformations). The vector-valued function $f()$ could be considered, in general, to be unknown although this is not our case since in this paper we consider the analytic solution provided by Mogi or Okada to solve the direct calculus represented by Eq. (15). However it is assumed, at least, that a set of pairs $\left(x_{i}, d_{i}\right)$, referred to as examples or learning patterns in typical neural network terminology, describing our knowledge about the function $f()$ are given.

The inverse problem consists of constructing a map which produces the vector $\boldsymbol{x}$ in response to $\boldsymbol{d}$ :

$\boldsymbol{x}=f^{-1}(\boldsymbol{d})$,

where the vector-valued function $f^{-1}$ denotes the inverse of $f$. The inverse function $f^{-1}$ is of course unknown and in most cases, including the one considered in this paper, $f^{-1}$ does not exist in closed form. Thus it is not possible to compute directly the vector $\boldsymbol{x}$ corresponding to a $\boldsymbol{d}$ data set. Some authors (see Nunnari et al., 2001 for instance) have shown that in case of ground deformation problems $f^{-1}$ can be approximated by using Multi-layer Perceptron (MLP) neural networks. However, in this paper we have chosen to avoid this inversion scheme and adopt a strategy based on the use of search algorithms. Indeed, although optimization strategies are more expensive in terms of computational effort they are more accurate (Nunnari et al., 2001).

\subsection{Inversion by using Genetic Algorithms}

In this paper we have considered an optimization approach based on the use of the Genetic Algorithms (Goldberg, 1989) to deal with the inverse problem. GAs implement optimization strategies based on the simulation of natural laws in order to obtain the fittest individual in the evolutionistic sense. Adopting this analogy, the optimal solution corresponds to the fittest individual. GAs search for the best value of the function to be optimised starting from a "population" of points belonging to the function domain (not from a single one). This reduces the probability of finding local minima. Moreover, GAs do not require the first derivative knowledge of the objective function or of other auxiliary information. Finally, GAs use probabilistic transition rules during iteration. Adopting a natural analogy, the variables involved in the optimisation, are codified in a particular structure similar to a chromosomal one. For example, a parameter can be translated into a string of elements (bit digits) which will be manipulated by appropriate operators during the evolution of the algorithm. The basic string operators to be applied are as follows.

- Reproduction: consists of duplicating a string.

- Crossover: given two different strings, the operator consists of exchanging substrings defined by some randomly chosen markers.

- Mutation: a variation of a randomly chosen bit belonging to a selected string.
The reproduction operator is used to improve the number of fittest individuals in the population, the crossover operator to recombine genetic information between different parents, and the mutation operator to introduce new information into the knowledge base. Each string is characterized by a real value named "fitness," strictly connected to the function that has to be optimized and used to select the more promising elements of the population. The strings applied by the operators are chosen according to their fitness. The higher the fitness function, the closer a point belonging to the considered domain will be to the optimal minimum/maximum. Therefore, the fitness value is fundamental to single out the more promising individuals of the population.

The suitability of ground deformation inversion scheme based on the use of GA has been assessed by various authors (such as Fernandez et al., 2001; Sambride and Mosegaard, 2002; Tiampo et al., 2004).

One of the problems arising on using optimization strategies is the choice of an appropriate cost function, more often referred to, among GA practitioners, as the fitness function. It is to stress here that there are no specific rules to make this choice and in general each author proposes his own fitness function. For instance, Tiampo et al. (2004) for solving a ground deformation problem similar to the one considered in this paper, propose the use of a fitness function based on the chi-square index. We have restricted our possibilities to two different definitions. The first is the traditional least square error, given by expression (17). In this expression $N$ represents the number of measuring points while $O_{i}$ and $P_{i}$ indicate the expected (observed) and simulated values, respectively.

$J=\sum_{i=1}^{N} \sqrt{\left(O_{i}-P_{i}\right)^{2}}$.

The second type of cost function considered is given by Eq. (18):

$J=\frac{\sum_{i=1}^{N}\left(P_{i}-O_{i}\right)^{2}}{\sum_{i=1}^{N}\left(\left|P_{i}-\bar{O}\right|+\left|O_{i}-\bar{O}\right|\right)^{2}}$.

Looking at expression (22) given below, it appears that minimizing of Eq. (18) reflects in the maximization of a performance index usually referred to as the index of agreement, which gives a relative measure of the degree of which predictions are error-free. The denominator of expression (18) accounts for the model's deviation from the mean of the observations as well as to the observations deviation from their mean. It does not provide information regarding unsystematic errors. With respect to a good model the index of agreement (22) should approach one. We have no theoretical results to say if cost function (17) works better than Eq. (18) but from several trials we are convinced that expression (17) gives better results when $N$ is relatively small (e.g. $N=30$ ) while expression (18) is more appropriate for larger $N$. 
Table 1. Performance indices computed to evaluate the error related to the GAs inverse modelling approach.

\begin{tabular}{lrccc}
\hline \multicolumn{1}{c}{ Parameter } & \multicolumn{1}{c}{ Bias } & RMSE & NMAE $\%$ & $d$ \\
\hline Dip angle $^{\circ}$ & -0.2533 & 0.3136 & 0.2815 & 0.9999 \\
Strike angle $^{\circ}$ & -0.2435 & 0.3215 & 0.0676 & 0.9997 \\
Length (m) & 0.0177 & 0.0247 & 0.2952 & 0.9965 \\
Width (m) & 0.0027 & 0.0155 & 0.3291 & 0.9978 \\
Strike Slip (m) & -0.0642 & 0.1561 & 3.4742 & 0.9990 \\
Dip Slip (m) & 0.1998 & 0.2117 & 4.9946 & 0.9904 \\
Opening (m) & -0.0048 & 0.0210 & 0.5161 & 0.9972 \\
Depth (m) & 0.1206 & 0.1330 & 3.0139 & 0.9956 \\
Xs (m) & -0.1455 & 0.1822 & 0.7275 & 0.9877 \\
Ys (m) & 0.0178 & 0.2831 & 1.3329 & 0.9994 \\
\hline
\end{tabular}

3.3 The experimental inversion scheme and error evaluation

In order to assess that the direct model is invertible and estimate the accuracy, one hundred models uniformly distributed in the space of parameters were inverted, hypothesizing a grid with $21 \times 21$ vertices (i.e. 441 measuring points). Furthermore, to evaluate the error related with the considered inverse modelling approach, an appropriate number of performance indexes have been computed, including the Bias (19), the RMSE (20), the NMAE\% (Percentage Normalised MAE) (21) and the index of agreement $d$ (22) .

BIAS : $\quad \frac{1}{N} \sum_{i=1}^{N}\left(P_{i}-O_{i}\right)$

RMSE $: \quad \sqrt{\frac{1}{N} \sum_{i=1}^{N}\left(P_{i}-O_{i}\right)^{2}}$

NMAE $\%=\frac{100}{(N \cdot \text { Range })} \sum_{i=1}^{N}\left|P_{i}-O_{i}\right|$

$d: \quad 1-\frac{\sum_{i=1}^{N}\left(P_{i}-O_{i}\right)^{2}}{\sum_{i=1}^{N}\left(\left|P_{i}-\bar{O}\right|+\left|O_{i}-\bar{O}\right|\right)^{2}}$.

In the expressions above, the over bar indicates the mean value while the suffix $i$ represents the generic value. The results obtained are shown in Table 1.

It can be seen that all the model parameters are estimated with an NMAE\% that is lower than 5\% in the case of freenoise data. In more detail, the source coordinates, the "Dip" and "Strike" angles, the "Length" and "Width" of the fault and the opening dislocation component $U_{3}$ are obtained with a NMAE $\%$ lower than $1 \%$, the coordinates $X_{s}$ and $Y_{S}$ exhibit NMAE\% lower than $2 \%$ and finally the "Depth" and the $U_{1}$ (Strike Slip) and $U_{2}$ (Dip Slip) dislocation components exhibit NMAE\% lower than 5\%. No appreciable differences

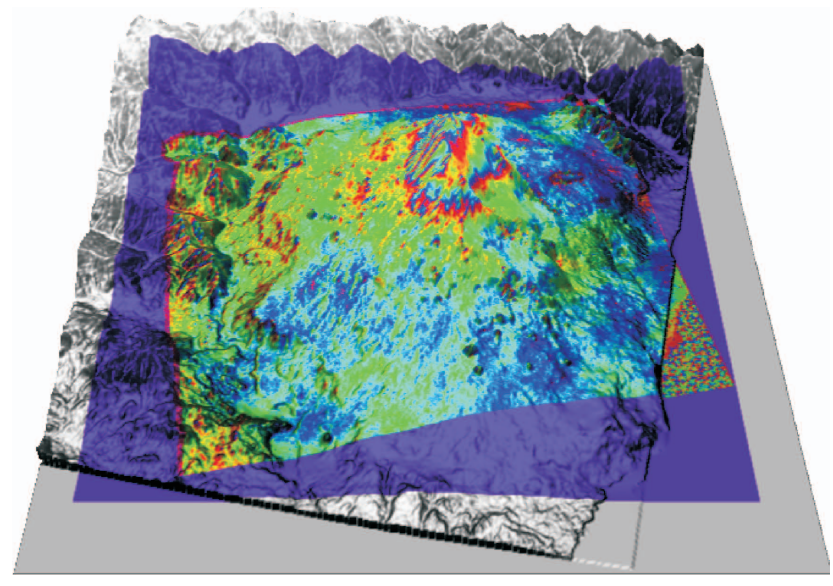

Fig. 4. Differential interferogram in phase $(-\pi \pi)$ relevant to Mt. Etna area referring to the ascending pair 22 July-26 August 1998.

were observed by using expression (18) as cost function. The results obtained show that the considered inverse problem can be solved with high accuracy in the case of free noise synthetic data, in the hypothesis that the deformation is due to a single dislocation.

\section{Inversion of actual data}

In order to test the algorithm with actual data, we have considered two eruptive events occurring at Mt. Etna during 1998 and 2001, respectively. The first eruptive event is used to test the Mogi source model and the second for the Okada source model.

4.1 Modelling a deformation pattern recorded during 1998 at Mt. Etna

A vigorous explosive eruption was produced by the Mt. Etna Voragine Crater on 22 July 1998. A $10 \mathrm{~km}$ high eruptive column above the crater rim formed at the eruptive climax between 16:48 and 17:14 GMT (Aloisi et al., 2002).

Fieldwork measurements, grain size analysis and calculation of the physical parameters allowed to characterize the eruption that proved to be of sub-plinian type. The volume of material erupted was estimated to be about $10^{6} \mathrm{~m}^{3}$ plus $2 \times 10^{6} \mathrm{~m}^{3}$ of proximal deposit ${ }^{1}$. Analysis of the deformation patterns associated with the Mt. Etna paroxysmal event of 22 July 1998, exhibits a positive elevation trend in the summit area of the volcano. In more detail, an uplift was observed in the pre-event period followed by a short lowering phase and hence a reprise of the uplift ${ }^{2}$. The short post event period,

\footnotetext{
${ }^{1}$ Andronico, D., Coltelli, M., and Del Carlo, P.: Tephra fallout from the smaller documented Subplinian eruption occurred at Mt. Etna on 22 July 1998, in preparation.

${ }^{2}$ Coltelli, M., Puglisi, G., Guglielmino, F., and Palano, M.: Application of differential SAR interferometry for studying eruptive event of 22 July 1998 at Mt. Etna, submitted on Quaderni di Geofisica, submitted.
} 
Table 2. Inversion results of the of the ground deformation recorded between 22 July-26 August 1998 by InSar using a Mogi type source.

\begin{tabular}{lccc}
\hline \multicolumn{1}{c}{ Searched parameter } & Returned value & Minimum & Maximum \\
\hline$-2 \mathrm{C}\left(\mathrm{GPa}^{*} \mathrm{~m}^{3}\right)$ & -47600000 & -80000000 & -10000000 \\
$f-$ Depth to reference level $(\mathrm{m})$ & 3151 & 10 & 3200 \\
$X_{S}-$ East coordinate $(\mathrm{m})$ & 500316 & 494000 & 504000 \\
$Y_{S}-$ North coordinate $(\mathrm{m})$ & 4178936 & 4172500 & 4184500 \\
\hline
\end{tabular}

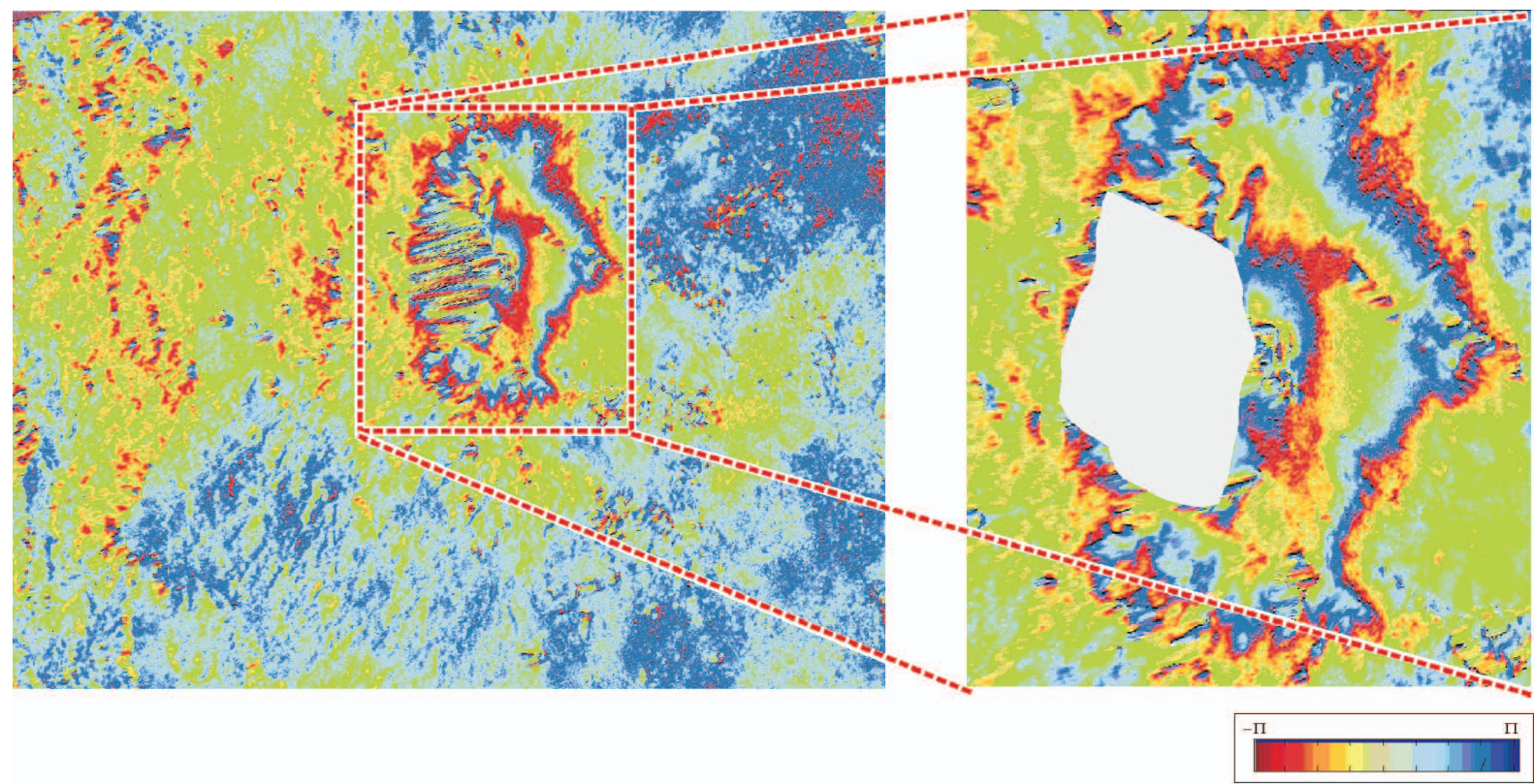

Fig. 5. (Left) Geocoded ascending interferogram refers to image pair 22 July-26 August 1998 and (right) considered detail (phase). The blank area shows the effect of a filter applied to mask the geometric errors that typically affect SAR interferograms.

to which the image pair 22 July-26 August 1998 refers as shown in Fig. 4, will be the object of the inversion exercise reported below.

Since the larger deformation effect is visible at the center of the image, i.e. the area just beneath the central craters, it was decided to use only a portion of the whole SAR image. This allows simplifying the inversion process and, at the same time, reducing the computational effort. The considered detail image is shown in Fig. 5.

The inversion approach described in the previous Sect. 3 was considered to find the optimal parameters of a Mogi type source and the results obtained are shown in Table 2.

Results show that the inversion algorithm returned the source coordinate (East and North) with a good accuracy, since these values strictly agree with other geological evidences. The two remaining parameters, i.e. $-2 C=a^{3} \Delta P$, related with the nucleus strain according to expression (6) and the depth $f$, seem to be over estimated if compared with the source models proposals for this eruptive event (Coltelli et al., submitted ${ }^{2}$ ), probably due to the topographic effects that were not taken into account during the inversion process.
Work is in progress to overcome this drawback.
4.2 Modelling an intrusive episode recorded during 2001 at Mt. Etna

In order to test the inversion algorithm with the Okada model, a descending interferogram (Fig. 6a) referring to the image pair 15 November 2000-31 October 2001 of Mt. Etna area was considered. The low quality of the SAR image suggested using a resized area (Fig. 6b) characterized by high coherence.

This allowed obtaining a good map of displacement vectors along the satellite LOS. As for the synthetic data inversion, we have considered the case of a tensile dislocation; therefore the Strike-slip $\left(U_{1}\right)$ and Dip-slip $\left(U_{2}\right)$ dislocation components were forced to zero, while the remaining parameters were assumed to be searched within their physical ranges. The search process was stopped when the fitness (Eq. 17) and the index of agreement (Eq. 18) (both assumed in the cost function to be minimized) approached the level of 99\%. The results of the Genetic Algorithm search are shown in the first column of Table 3 . 
Table 3. Inversion results using independent SAR and GPS data.

\begin{tabular}{lcc}
\hline Searched parameter & Inversion with SAR data & Inversion using GPS data \\
\hline Lat. UTM $(\mathrm{km})$ & 500.77 & 500.65 \\
Long UTM $(\mathrm{km})$ & 4176.275 & 4175.5 \\
Azimuth & & 3.4 \\
Depth $(\mathrm{km})$ & 1.3 & 1.6 a.s.l. \\
Length $(\mathrm{km})$ & 1.5 a.s.l. & 2.34 \\
Width $(\mathrm{km})$ & 7.4 & 3.55 \\
Dip $^{\circ}$ & 1.5 & 89 \\
Opening $(\mathrm{m})$ & 89.9 & 2.51 \\
\hline
\end{tabular}

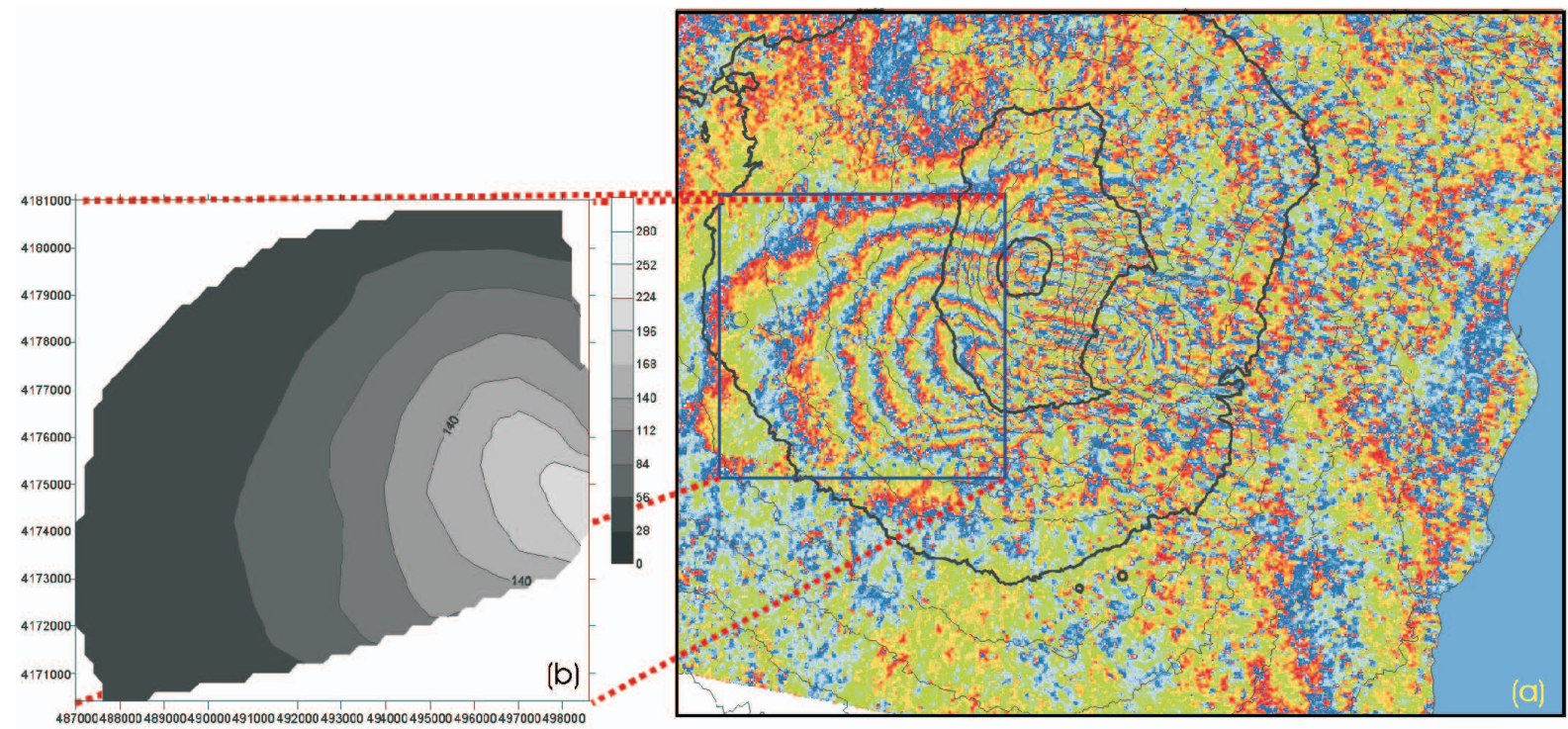

Fig. 6. (a) Geocoded descending interferogram referring to the image pair 15 November 2000-31 October 2001 of the Mt. Etna area, (b) geocoded LOS displacement map (expressed in $\mathrm{mm}$ ) of resize area.

In order to assess the reliability of the returned parameters, an independent inversion process was performed by using a set of ground deformation data recorded during two consecutive surveys performed in July 2001 and September 2001. Results returned by the inversion algorithm referring to this GPS data set are shown in the last column of Table 3. The comparison between SAR and GPS results shows a high agreement among the Latitude, Longitude, Azimuth, Depth, Dip and Opening parameters. On the other hand, it is possible to observe a significant difference between the Length and Width parameters in the two inversion exercises. The authors have tried to interpret the differences concerning these two model parameters by invoking the fact that the SAR and GPS data sets were recorded in different time intervals. Indeed, the SAR available data set refers to a time interval spanning from November 2000 to October 2001, while the GPS data was recorded during July-September 2001. This means that the SAR data set probably reflects the presence of a deep volume in the basement, beneath the west flank of the volcano, that was progressively pressurizing before the eruption and quickly depressurizing during the eruption.
However, the parameters obtained for the dyke are globally consistent with other geological evidences and with results reported in other papers concerning the 2001 Etna eruption such as Bonaccorso et al. (2002).

\section{Conclusions}

In this paper a method for the inversion of SAR interferometric data by using a GA optimization algorithm has been proposed. More specifically, it has dealt with the inversion of ground deformation data relating to hydrostatically pumped spherical sources (Mogi) and magma-filled dikes (Okada) that are often considered to fit ground deformation data observed in active volcanic areas such as Mt. Etna. The goodness of the inversion procedure has been assessed both using synthetic and actual SAR data.

The inversion of synthetic data has shown a high reliability of the proposed approach, thus demonstrating that the inverse problem is well conditioned. As was expected, the inversion of actual SAR interferograms is more difficult than 
synthetic data. Indeed, actual data are affected by a number of noise effects. Furthermore, topographic effects in the area under consideration should be adequately considered, since the real ground behaves differently to the flat, homogenous and isotropic half space considered as the base for both Mogi and Okada models. Another relevant problem which has not yet been taken into account is the possibility of multiple sources acting at the same time. From a methodological point of view, there are no particular problems for GA optimization algorithm to search for more than one source at the same time. However, it is still not known if the inverse problem in the presence of multiple sources is well conditioned. Work is in progress to overcome all these shortcomings.

Acknowledgements. The manuscript has benefited from the insightful reviews by J. Fernández, and the authors have appreciated his helpful comments. This work has been supported by the Italian GNV (Gruppo Nazionale per la Vulcanologia) under the coordinated project "Sviluppo ed Applicazione di Tecniche di Telerilevamento per il Monitoraggio dei Vulcani Attivi Italiani". The ERS data were provided in the frame of the ERS AO3-359 project.

Edited by: G. Zöller

Reviewed by: J. Fernandez

\section{References}

Aloisi, M., D’Agostino, M., Kenneson, G .D., Mostaccio, A., and Neri, G.: Satellite analysis and PUFF simulation of the eruptive cloud generated by Mt. Etna paroxism of 22 July 1998, J. Geophys. Res., 107, 1-12, 2002.

Bonaccorso, A., Aloisi, M., and Mattia, M.: Dike emplacement forerunning the Etna July 2001 eruption modelled through continuous tilt and GPS data, Geophys. Res. Lett., 29, 13, 2002.

Bonaccorso, A., Calvari, S., Coltelli, M., and Falsaperla, S.: Mt. Etna: Volcano laboratory, AGU Geophysical Monography, 143, 1-369, 2004.

Briole, P., Avallone, A., Beauducel, F., Bonforte, A., Cayol, V., Deplus, C., Delacourt, C., Froge, J. L., Malengreau., B., and Puglisi, G.: Interferometrie Radar Appliquée aux Volcans: Cas de L'Etna et des Champs Phlégréens (Italie), Rapport Quadriennal CNFGG 1995-1998, pres. at Union Internationale de Geodesie et Geophysique, Birmingham, 121-128, 1999.

Bürgmann, R., Rosen, P. A., and Fielding, E. J.: Synthetic aperture radar interferometry to measure Earth's surface topography and its deformation, Ann. Rev Earth Planet. Sci. 28, 169-209, 2000.

Coltelli, M., Dutra, L., Fornaro, G., Franceschetti, G., Lanari., R., Migliaccio, M.,. Moreira, J. R., Papathanassiou, K.P., Puglisi, G., Riccio, D., and Schwäbisch, M.: SIR-C/X-SAR Interferometry over Mt. Etna: DEM Generation, Accuracy Assessment and Data Interpretation, DLR-Forschungsbericht, 95-48, 1996.

Gabriel, A. K., Goldstein, R. M., and Zebker, H. A.: Mapping Small Elevation Changes over Large Areas: Differential Radar interferometry, J. Geophys. Res., 94, B7, 9183-9191, 1995.
Fernández, J., Tiampo, K. F., Jentzsch, G., Charco, M., and Rundle, J. B.: Inflation or deflation? New results for Mayon volcano applying elastic-gravitational modelling, Geophys. Res. Lett., 28, 2349-2352, 2001.

Fernández, J., Yu, T. T., Rodriguez-Velasco, G., GonzálezMatesanz, J., Romero, R., Rodríguez, G., Quirós, R., Dalda, A., Aparicio, A., and Blanco, M. J.: New geodetic monitoring system in the volcanic island of Tenerife, Canaries, Spain. Combination of InSAR and GPS techniques, J. Volcanol. Geotherm. Res., 124/3-4, 241-253, 2003.

Goldberg D. E.: Genetic Algorithms in Search, Optimization and Machine Learning, Reading, MA, Addison-Wesley, 1989.

Güdmundsson, S., Sigmundsson, F., and Carstensen, J. M.: Threedimensional surface motion maps estimated from combined interferometric synthetic aperture radar and GPS data, J. Geophys. Res., 107, B10, 2250, doi:10.1029/2001JB000283, 2002.

Lanari, R., Fornaro , G., Riccio, D., Migliaccio, M., Papathanassiou, K. P., Moreira, J. R., Schwäbisch, M., Dutra, L., Puglisi, G., Franceschetti, G., and Coltelli, M.: Generation of Digital Elevation Models by Using SIR-C/X-SAR Multifrequency TwoPass Interferometry: The Etna Case Study, IEEE Trans. on Geoscience and Remote Sensing, 34, 5, 1097-1114, 1996.

Massonet, D. and Feigl, K. L.: Radar interferometry and its application to changes in the eartth's surface, Rev. Geophys., 36, 441-500, 1998.

Massonnet, D. and Rabaute, T.: Radar Interferometry: Limits and Potential, IEEE Trans. on Geoscience and Remote Sensing, 31, 2, 435-464, 1993.

McCloy, K. R.: The physical principles of remote sensing, Resource Management Information System - Process and Practice, Taylor \& Francis press, 2, 67-96, 1995.

Mogi, K.: Relation between the eruptions of various volcanoes and the deformation of ground surfaces around them, Bulletin of the Earthquake Research Institute (University of Tokyo), 36, 99 134, 1958.

Nunnari, G., Bertucco, L., and Ferrucci, F.: A Neural Approach to the Integrated Inversion of Geophisical Data Types, IEEE Transaction on Geosciences and Remote Sensing, 39, 4, 736-748, April 2001.

Omori, F.: The Sakura-jima eruptions and earthquakes, Bulletin of the Imperial Earthquake Investigation Committee, 8, 1-6, 1914.

Okada, Y.: Surface deformation due to shear and tensile faults in a halfspace, Bull. Seismol. Soc. Amer., 75, 1135-1154, 1985.

Puglisi, G. and Coltelli, M.: Sar interferometry applications on active volcanoes: state of the art and perspectives for volcano monitoring, Il Nuovo Cimento, 24C, 133-145, 2001.

Sambride, M., and Mosegaard, K.: Montecarlo Methods in Geophisical inverse problems, Rev. Geophys., 40, 3, doi:10.1029/2000RG000089, 2002.

Sasai, Y.: Tectonomagnetic modelling on the basis of the linear piezomagnetic effect, Bullettin of the Earthquake Research Institute, University of Tokio, 66, 585-722, 1991.

Tiampo, K. F., Fernandez, J., Gentzsch, G., Charco, M., and Rundle, J. B.: Inverting for the parameters of a volcanic source using a genetic algorithm and a model for magmatic intrusion in elastic-gravitational layered Earth models, Computer \& Geosciences, 30/9-10, 985-1001, 2004. 